OPEN ACCESS

Edited by:

Markus Roth,

Albert Ludwigs Universität Freiburg,

Germany

Reviewed by:

Giuseppe Bono,

University of Rome Tor Vergata, Italy Hilding Neilson,

University of Toronto, Canada

*Correspondence:

Nancy Remage Evans nevans@cfa.harvard.edu

Specialty section:

This article was submitted to Stellar and Solar Physics,

a section of the journal

Frontiers in Astronomy and Space

Sciences

Received: 07 February 2019 Accepted: 24 September 2019

Published: 24 October 2019

Citation:

Evans NR and Engle S (2019)

Prospects for X-Ray and FUV

Observations to Provide Insight Into

the "Cepheid Mass Problem".

Front. Astron. Space Sci. 6:65.

doi: 10.3389/fspas.2019.00065

\section{Prospects for X-Ray and FUV Observations to Provide Insight Into the "Cepheid Mass Problem"}

\author{
Nancy Remage Evans ${ }^{1 *}$ and Scott Engle ${ }^{2}$ \\ ${ }^{1}$ High Energy Astrophysics Division (HEAD), Smithsonian Astrophysical Observatory (SAO), Cambridge, MA, United States, \\ ${ }^{2}$ Department of Astronomy and Astrophysics, Villanova University, Villanova, PA, United States
}

The classical Cepheid Leavitt Law (Period-Luminosity relation) has been known for 100 years, and used in extragalactic distance determination for nearly as long. Cepheids are particularly important in testing the inferences of asteroseismology, and the comparison of masses inferred from pulsation calculations, evolutionary calculations, and observationally measured masses is termed the "Cepheid mass problem." However, Cepheids still present us with surprises. Recent results about X-ray behavior of $\delta$ Cep is one example. At minimum radius the pulsation wave from the envelope creates a disruption in the photosphere and chromosphere. X-ray activity, on the other hand remains constant at a low level at this phase. Just after maximum radius, however, $\mathrm{X}$-ray flux increases sharply (4-fold in the case of $\delta$ Cep). We summarize here the observational program to explore parameters involved and theoretical investigations searching for a basic understanding of this upper atmosphere phenomenon. This results in new diagnostics of the linkages between the pulsating envelope, the upper atmosphere, and possible mass-loss in the system.

Keywords: Cepheids, variable stars, X-rays, Leavitt Law, distance scale

\section{INTRODUCTION}

Classical Cepheid variable stars are reasonably massive stars (typically $5 \mathrm{M}_{\odot}$ ) which pulsate radially with periods of about a week. The classical Cepheid Leavitt Law (Period-Luminosity relation) has been known for more than 100 years, and it is an important step in determining extragalactic distances. The Leavitt Law is arguably the first case of asteroseismology, since the relation between brightness and period was the first indication of the phenomenon. While Cepheids are young (typically $50 \mathrm{Myr}$ old), they have evolved beyond the main sequence and are burning $\mathrm{He}$ in the core. Along the instability strip, classical Cepheids span a range of periods of 2 to $>20$ days, masses of 3 to $8 \mathrm{M}_{\odot}$ and ages of 30 to at least $130 \mathrm{Myr}$ (Bono et al., 2005; Anderson et al., 2014), with additional scatter due to pulsation mode, metallicity, and rotation.

Fifty years after the discovery of the Leavitt Law the mechanism of pulsation was determined and hydrodynamic calculations (e.g., Christy, 1966; Cox et al., 1966) led to the derivation of masses. Comparison of these masses with masses inferred from evolutionary tracks disagreed, with the evolutionary masses being larger (termed "the Cepheid mass discrepancy"). Recent summarizes are provided by Neilson et al. (2011) and Anderson et al. (2014). Internal physics which has been investigated to explain this includes opacity (revisions considerably reduced the discrepancy), core convective overshoot on the main sequence, mass loss, and rotation. Although this question has not been fully answered, the discrepancy is now 10 to $20 \%$ (Neilson et al., 2011) and rotation in models (Anderson et al., 2014, 2016) may reduce it further. 
The Cepheid Leavitt Law is still the foundation of the extragalactic distance scale. Figure $\mathbf{1}$ below provides an example of the extensive observational data set which as been built up, including photometry and spectroscopy in optical, ultraviolet, $\mathrm{X}$-ray wavelengths, as well as near and far infrared (IR). Today, use of the IR version of the Leavitt Law is favored, particularly because of the decreased sensitivity of this region to reddening (Freedman and Madore, 2010).

However, there have recently been several features related to the atmosphere above the photosphere which raise questions about our understanding of these upper regions. They pertain to the question of whether Cepheids are losing mass, which relates to the problem of using evolutionary tracks to interpret these stars. Specifically, infrared and visible shells (circumstellar envelopes or CSEs) are observed in interferometric observations (Mérand et al., 2015; Nardetto et al., 2016). The present discussion summarizes new observations of the upper atmosphere in X-rays and ultraviolet which provide crucial clues to linkages between stellar parameters (mass, temperature, luminosity) and processes such as subtle mass loss which may influence our interpretation and calculations. Furthermore, the CSEs may affect the IR Leavitt Law, and hence distance calculations.

Mass loss has been notoriously difficult to measure in Cepheids since it is small and the stars are variable. Measurements of mass loss have been made from data from the IRAS and Spitzer satellites in the IR, and the IUE satellite in the ultraviolet as summarized by Marengo et al. (2010). The estimates vary widely $\left(10^{-10}\right.$ to $10^{-7} \mathrm{M}_{\odot} \mathrm{yr}^{-1}$ and higher), with large uncertainties. One of the goals of understanding the upper atmosphere using the new diagnostics of CSEs and $\mathrm{X}$-rays is to quantify their relation to mass loss and ultimately to determine whether mass loss is an important component of the Cepheid phase.

Observationally there are now measured masses for several Milky Way Cepheids (summarized by Evans et al., 2018a). Of particular importance is the use of interferometry to determine a very accurate mass for V1334 Cyg (Gallenne et al., 2018), which is markedly smaller than the evolutionary mass produced by several codes (Engle, 2019 private communication). In addition, masses for several Cepheids in eclipsing binaries in the LMC have been determined (Pilecki et al., 2018), making comparisons possible at two metallicities. We note that Gaia observations will provide major contributions to distances as well as orbits and masses. However, because of the prevelance of binaries among Cepheids, these improvements will not be fully realized until the DR3 release which incorporates orbital solutions.

The continuing development of theoretical and measured masses is a good example of the effort required to confirm inferences from asteroseismology. At present, in particular, long strings of photometry from satellites permit new insights into the interiors of many variable stars. The "Cepheid mass problem" is a prime example of the need for observational confirmation to improve our understanding of these cornerstone stars.

\section{MULTIWAVELENGTH OBSERVATIONS}

To understand the outer layers of Cepheids, a series of multiwavelength observations is underway as part of the program "The Secret Lives of Cepheids" (Engle, 2015). For the prototype $\delta$ Cep itself these are summarized in Figure 1 (reprinted from Engle et al., 2017). Far ultraviolet observations starting with the International Ultraviolet Explorer (IUE) (Schmidt and Parsons, 1982) have shown that many high temperature lines above the photosphere go into emission at some phases. Because of the low scattered light background, the Cosmic Origins Spectrograph (COS) on the Hubble Space Telescope (HST) has been particularly effective in carrying out far ultraviolet observations of Cepheids. As an example for $\delta$ Cep, the emission strengths of two lines are shown in the top two panels in Figure 1, together with photometry, radial velocities, and radius variations around the pulsation cycle. Three separate aspects of the HST-COS data offer a strong confirmation of the long-held view that pulsation-driven shock waves propagate through the atmospheres of Cepheids.

First, the rapid increase in emission line flux occurs shortly after minimum radius (i.e., when the Cepheid photosphere begins to expand again). This is the phase at which the shock would emerge from the photosphere and propagate through the chromosphere.

Second, the line profiles continually broaden during the phases of increasing and maximum emission (e.g., see Figure 2). Radial velocity measures at these phases show the photosphere to be shrinking while the line-emitting chromosphere is expanding. This compression and thermal excitation of the atmosphere is responsible for the line broadening. In addition, at pulsation phase $\phi=0.04$ for $\delta \mathrm{Cep}$, the line intensity is decreasing from maximum yet the line profile is the broadest of all observations. This indicates that we are observing a highly turbulent, postshock chromosphere at this phase.

Finally, at all phases of increased emission, an additional blueshifted component (see Figure 2) becomes visible in the warm far ultraviolet (FUV) lines. This component indicates the presence of excited plasmas with a small range in velocity (directly affected by the passing shock) as compared with the broad profiles from the turbulent post-shock.

Though the FUV observations point to a consistent picture, $\mathrm{X}$ ray data tell a different story. Using observations from Chandra and $X M M-N e w t o n$, during the period before and after minimum radius X-ray activity remains at a constant low level. Just after maximum radius there is a burst ( $\simeq 4$-fold increase) of activity, as shown in the third panel in Figure 1. This activity has been confirmed in an additional cycle for $\delta$ Cep (Engle et al., 2017). This behavior has also been seen in the $9.8^{d}$ Cepheid $\beta$ Dor (Engle, 2019 private communication). In $\beta$ Dor the phase is somewhat different when calculated from the standard ephemeris (based on maximum light). However, the period of $\beta$ Dor is $9.8^{d}$, within the period range in the Hertzsprung progression where the pulsation amplitude is decreased by the coincidence of the primary and secondary humps at maximum light. On the other hand, if we use phase of increased chromospheric emission (minimum radius) as a fiducial the phase of X-ray increase has 


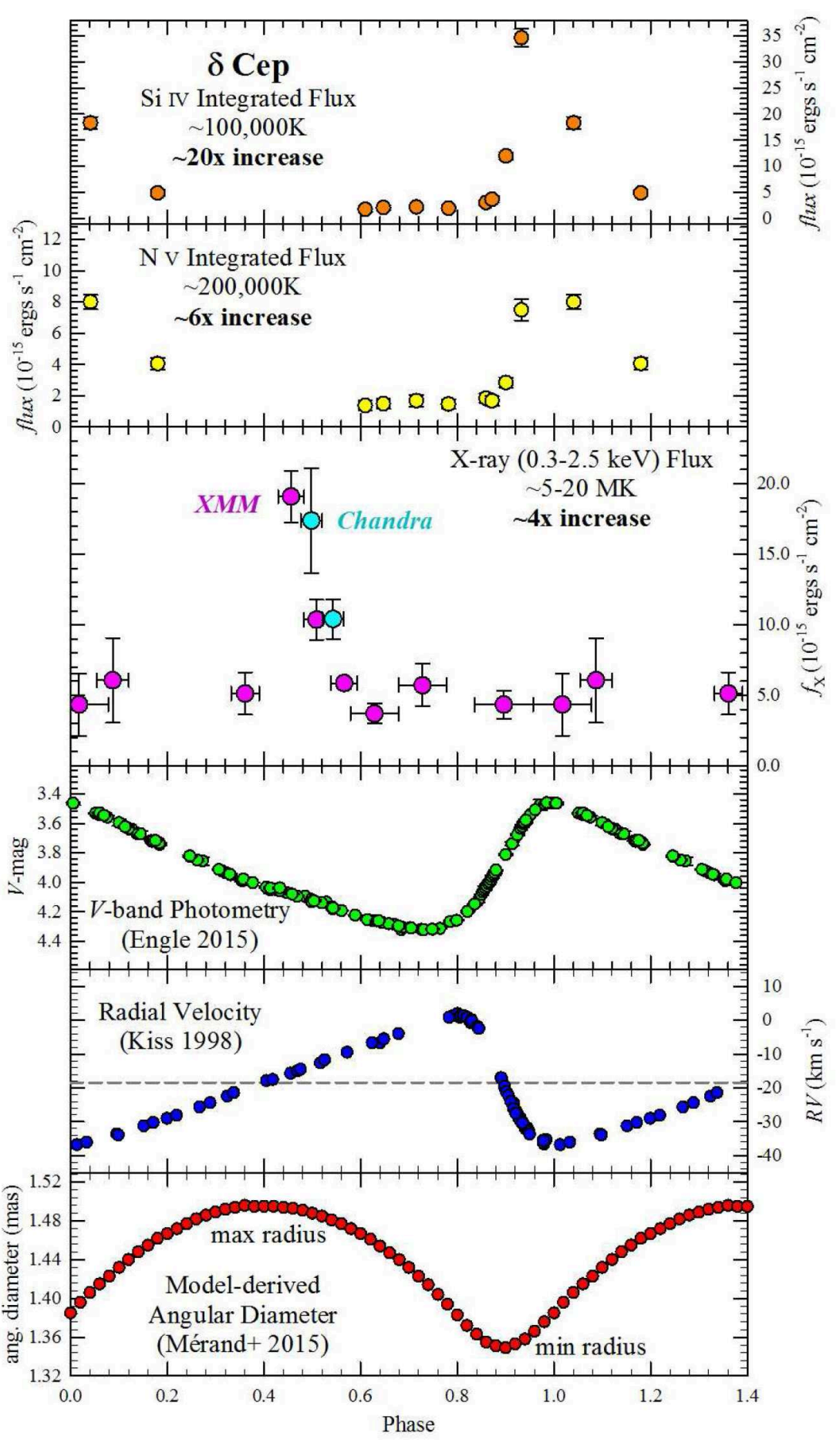

FIGURE 1 | Atmospheric variations of $\delta$ Cep as a function of pulsation phase. The top 2 panels show FUV emission line fluxes. The middle panel shows X-ray flux: pink points: XMM data from several cycles; cyan points: Chandra data from the same cycle. The bottom three panels show the $\mathrm{V}$ light curve, the radial velocity curve and the radius curve. Reprinted from the Astrophysical Journal (Kiss, 1998).

the same relation to minimum radius as $\delta$ Cep (Evans et al., $2018 \mathrm{~b})$. This implies that the same mechanism is working in both stars. There is an additional star with a tentative X-ray increase at the phase of maximum radius. Böhm-Vitense and Parsons (1983) found a tentative detection of $\zeta$ Gem (alone among the Cepheids they observed) with the Einstein satellite 


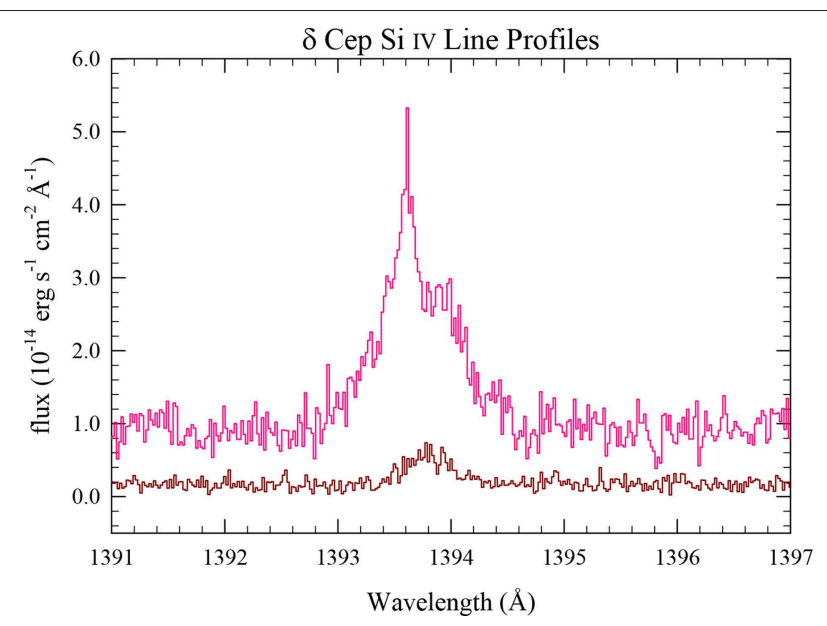

FIGURE 2 | The Si IV 1394^ emission line profile of $\delta$ Cep is plotted at two representative phases. The bottom spectrum was taken at $\phi=0.86$, and the top spectrum at $\phi=0.93$. The increased activity level and overall broadening of the emission line are visible, along with the additional blue-shifted emission component in the top spectrum.

at the phase expected from the $\beta$ Dor observations, again at maximum radius.

We characterize the X-ray variations as possibly being triggered by the collapse of the atmosphere following maximum radius, while apparently remaining unaffected by the disturbance in the photosphere and chromosphere at minimum radius. Similarly, spectra in visible wavelengths are indistinguishable from those of non-variable supergiants at the phases of the X-ray burst. There seem to be two likely possibilities to account for the $\mathrm{X}$-ray burst. First, a shock wave from the pulsation cycle might generate velocities high enough to produce X-rays. This would require velocities higher than typically seen over the pulsation cycle in the photosphere (Figure 1). The second possibility is a "flare event" as seen on the sun, regulated by the pulsation cycle. Flares (reconnection events) require magnetic fields. However, stars with a convective surface typically have flares, and the occurrence of low level X-rays outside the phases of the burst in Cepheids is consistent with a magnetic field. Magnetic fields have been measured in the Cepheid $\eta$ Aql and the supergiant $\alpha$ Per (Grunhut et al., 2010). In the sun, flares are often followed by a coronal mass ejection (CME), which could be part of the overall mass-loss scenario that has been helping to sustain an infrared shell.

We have embarked on a program of X-ray observations to determine whether the occurrence and strength of an X-ray burst depends on the amplitude of pulsation, the luminosity (hence the mass) of the star, the temperature of the star (hence the

\section{REFERENCES}

Anderson, R. I., Ekström, S., Georgy, C., Meynet, G., Mowlavi, N., and Eyer, L. (2014). On the effect of rotation on populations of classical importance of convection), and the pulsation mode (hence the depth of the node in the envelop) using observations of Cepheids which span a range of these parameters.

The X-ray variations that we have observed remain puzzling. However, when Cepheids are placed within the broader context of cool supergiants, X-ray activity may be more common than originally thought. Recent studies (Ayres, 2017, 2018) have shown that a number of non-pulsating, cool supergiants also display FUV emission lines and X-ray activity. Using ratios of FUV/X-ray to bolometric luminosity, the studies show that Cepheids behave similarly to their non-pulsating counterparts, and stand apart from cool dwarf stars. The cool dwarfs consistently show relatively higher levels of FUV and X-ray activity, and the magnetic fields of cool dwarfs have also been well-studied and are considered nearly ubiquitous. So perhaps the pulsations of Cepheids play no role in generating FUV or $\mathrm{X}$-ray activity; they simply modulate what would have otherwise been persistent and steady activity levels.

\section{SUMMARY}

The emerging picture is a pattern of $\mathrm{X}$-ray bursts regulated by pulsation, which appear to be triggered by the collapse of the atmosphere after maximum radius. We are conducting a series of X-ray observations with Chandra and XMMNewton to determine the relation between the strength of X-ray increases and parameters such as pulsation amplitude and stellar luminosity (mass). We are investigating theoretical explanations of either a pulsation shock or a flare event. This pulsation related $\mathrm{X}$-ray activity appears to be a new ingredient possibly related to the outer atmosphere CSEs. Insight into the full picture of the outer atmospheres is important in the question of mass loss in Cepheids as well as the use of the infrared Cepheid Leavitt Law (Period-Luminosity relation).

The new diagnostics of the upper atmosphere of these pulsating stars in the ultraviolet and X-ray region will provide clues to the conundrum of the "Cepheid mass problem" which has challenged the interpretation of pulsation calculations (as well as evolutionary tracks) of Cepheids.

\section{AUTHOR CONTRIBUTIONS}

All authors listed have made a substantial, direct and intellectual contribution to the work, and approved it for publication.

\section{FUNDING}

This work was provided from the Chandra X-Ray Center NASA Contract NAS8-03060. 
analysis for metallicities $0.014,0.006$, and 0.002. Astron. Astrophys. 591:A8. doi: 10.1051/0004-6361/201528031

Ayres, T. (2017). A closer look at the alpha persei coronal conundrum. Astrophys. J. 837:14. doi: $10.3847 / 1538-4357 / 837 / 1 / 14$

Ayres, T. (2018). Cracking the conundrum of F-supergiant coronae. Astrophys. J. 854:95. doi: 10.3847/1538-4357/aaa6d7

Böhm-Vitense, E., and Parsons, S. (1983). EINSTEIN observations of three classical cepheids. Astrophys. J. 266:171.

Bono, G., Marconi, M., Cassis, S., Caputo, F., Gieren, W., and Pietrzynski, G. (2005). Classical cepheid pulsation models. X. The period-age relation. Astrophys. J. 621, 966-977. doi: 10.1086/4 27744

Christy, R. F. (1966). Pulsation theory. Annu. Rev. Astron. Astrophys. 4:353.

Cox, J. P., Cox, A. N., Olsen, K. H., King, D. S., and Eilers, D. D. (1966). Self-excited radial oscillations in thin stellar envelopes. Astrophys. J. 144:1038.

Engle, S. (2015). The Secret Lives of Cepheids: A Multi-Wavelength Study of the Atmospheres and Real-Time Evolution of Classical Cepheids (Ph.D. Thesis). Queensland: James Cook University.

Engle, S. G., Guinan, E. F., Harper, G. M., Cuntz, M., Evans, N. R., Neilson, H. R., et al. (2017). The secret lives of cepheids: $\delta$ Cep The prototype of a new class of pulsating X-ray variable stars. Astrophys. J. 838:67. doi: 10.3847/1538-4357/aa6159

Evans, N. R., Proffitt, C., Carpenter, K. G., Winston, E. M., Kober, G. V., Günther, H. M., et al. (2018a). The mass of the Cepheid V350 Sgr. Astrophys. J. 866:30. doi: $10.3847 / 1538-4357 /$ aade 03

Evans, N. R., Scott, E., Edward, G., Hilding, N., Massimo, M., Lynn, M., et al. (2018b). Outward from Cepheids. PTA 123:51.

Freedman, W. L., and Madore, B. F. (2010). The hubble constant. Annu. Rev. Astron. Astrophys. 48:672. doi: 10.1146/annurev-astro-082708-101829

Gallenne, A., Kervella, P., Evans, N. R., Proffitt, C. R., Monnier, J. D., Merand, A., et al. (2018). A geometrical 1 per cent Distance to the short-period binary Cepheid V1334 Cygni. Astrophys. J. 867:121. doi: 10.3847/1538-4357/aae373

Grunhut, J. H., Wade, G. A., Hanes, D. A., Alecian, E. (2010). Systematic detection of magnetic fields in massive, late-type supergiants. Mon. Notices R. Astron. Soc. 408:2290. doi: 10.1111/j.1365-2966.2010.17275.x
Kiss, L. L. (1998). A photometric and spectroscopic study of the brightest northern Cepheids - I. Observations. Mon. Notices R. Astron. Soc. 297:825.

Marengo, M., Evans, N. R., Barmby, P., Bono, G., Welch, D. L., Romaniello, M. (2010). Galactic Cepheids with Spitzer. I. Leavitt Law and colors. Astrophys. J. 709:120. doi: 10.1088/0004-637X/709/1/120

Mérand, A., Kervella, P., Breitfelder, J., Gallenne, A., Coude du Foresto, V., ten Brummelaar, T. A., et al. (2015). Cepheid distances from the SpectroPhoto- Interferometry of Pulsating Stars (SPIPS). Application to the prototypes $\delta$ Cephei and $\eta$ Aquilae. Astron. Astrophys. 584:A80. doi: 10.1051/0004-6361/201525954

Nardetto, N., Merand, A., Mourard, D., Storm, J., Gieren, W., Fouqué, P., et al. (2016). VEGA/CHARA interferometric observations of Cepheids. I. A resolved structure around the prototype classical Cepheid $\delta$ Cep in the visible spectral range. Astron. Astrophys. 593:A45. doi: 10.1051/0004-6361/2015 28005

Neilson, H. R., Cantiello, M., and Langer, N. (2011). The Cepheid mass discrepancy and pulsation-driven mass loss. Astron. Astrophys. 529:L9. doi: 10.1051/0004-6361/201116920

Pilecki, B., Gieren, W., Pietrzyński, G., Thompson, I. B., Smolec, R., Graczyk, D., et al. (2018). The Araucaria Project: high-precision Cepheid astrophysics from the analysis of variables in double-lined eclipsing binaries. Astrophys. J. 862:43. doi: 10.3847/1538-4357/aacb32

Schmidt, E. G., and Parsons, S. B. (1982). The chromospheres of classical Cepheids. I - Low resolution IUE spectra. Astrophys. J. 48:185.

Conflict of Interest: The authors declare that the research was conducted in the absence of any commercial or financial relationships that could be construed as a potential conflict of interest.

Copyright (c) 2019 Evans and Engle. This is an open-access article distributed under the terms of the Creative Commons Attribution License (CC BY). The use, distribution or reproduction in other forums is permitted, provided the original author(s) and the copyright owner(s) are credited and that the original publication in this journal is cited, in accordance with accepted academic practice. No use, distribution or reproduction is permitted which does not comply with these terms. 